\title{
Skull osteomyelitis as a rare complication of cat scratch disease
}

\author{
Yaseen Rafee ${ }^{1,2}$, B. Keith English ${ }^{3}$
}

${ }^{1}$ Department of Pediatrics, Division of Infectious Disease, Hurley Children's Hospital, Flint, Departments of ${ }^{2}$ Pediatrics and ${ }^{3} P e d i a t r i c s$ and Human Development, College of Human Medicine, Michigan State University, East Lansing, MI, USA

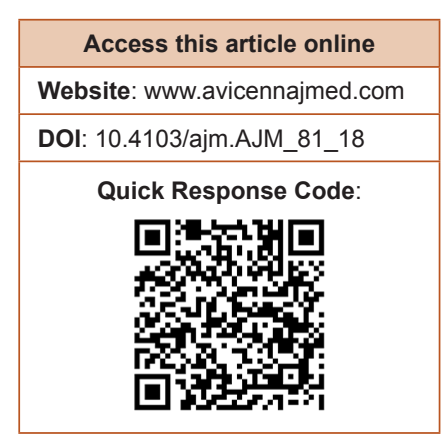

\begin{abstract}
Bartonella henselae, the causative agent of cat scratch disease (CSD), is one of the most common causes of regional lymphadenitis in children. Other less common manifestations of $B$. henselae infection including fever of unknown origin, neuroretinitis, and osteomyelitis are being increasingly recognized. We describe a 3-year-old female with a recent history of typical CSD involving lymph nodes who developed osteomyelitis of the skull, a very rarely recognized complication of this infection.
\end{abstract}

Key words: Bartonella henselae, cat scratch disease, osteomyelitis

\section{INTRODUCTION}

Bartonella henselae, the causative agent of cat scratch disease (CSD), is one of the most common causes of regional lymphadenitis in children, usually resulting in mild, self-limited infection. However, cat scratch disease has a broad spectrum of presentations in healthy children, including fever of unknown origin (with multiple hepatosplenic abscesses), neuroretinitis, osteomyelitis, and encephalitis. ${ }^{[1,2]}$ Serologic testing usually confirms the diagnosis of Bartonella infections, but both false positive and false negative results have been reported. New diagnostics, including nucleic acid amplification tests such as polymerase chain reaction (PCR), have allowed confirmation of $B$. henselae infection in patients with atypical manifestations. ${ }^{[3]}$ In this report, we describe a 3-year-old female with a recent history of typical CSD lymphadenitis who subsequently developed osteomyelitis of the skull, a very rarely recognized complication of this infection.

\section{CASE REPORT}

A 3-year-old Caucasian female who was recently diagnosed and treated for CSD presented to the emergency department

Address for correspondence: Dr. Yaseen Rafee,

Department of Pediatrics, Division of Infectious Disease,

Hurley Children's Hospital, Flint, MI, USA.

Department of Pediatrics, College of Human Medicine,

Michigan State University, East Lansing, MI, USA.

E-mail: yrafee1@hurleymc.org for a 2-week history of worsening scalp lump with redness. The lesion was in the left frontal region. She did not have a fever or any other systemic symptoms. Two weeks earlier, she finished a 5-day course of azithromycin for suspected CSD lymphadenitis. At that time, she had presented with right side inguinal and preauricular lumps, measuring $2 \mathrm{~cm}$ and $1 \mathrm{~cm}$, respectively. Both lumps were tender and erythematous. The patient had no significant medical history. Her growth and development were appropriate for her age. Her immunizations were complete for her age. She lived with her parents in Flint and had never traveled outside Michigan. The family had one dog and two kittens at home. Her mother reported that the kittens scratched the patient frequently.

On admission, the patient was alert, interactive, and in no distress. She was afebrile, and her vital signs were normal. Her examination was significant for a $4 \mathrm{~cm} \times 4 \mathrm{~cm}$ lump located in the left frontal area. There was mild erythema overlying the lesion, but it was not tender. There was a small area of fluctuance at the center. A $1 \mathrm{~cm} \times 1 \mathrm{~cm}$ preauricular lymph

This is an open access journal, and articles are distributed under the terms of the Creative Commons Attribution-NonCommercial-ShareAlike 4.0 License, which allows others to remix, tweak, and build upon the work non-commercially, as long as appropriate credit is given and the new creations are licensed under the identical terms.

For reprints contact: reprints@medknow.com

Cite this article as: Rafee $\mathrm{Y}$, English BK. Skull osteomyelitis as a rare complication of cat scratch disease. Avicenna J Med 2018;8:157-9. 
node was palpable on the right. A $2 \mathrm{~cm}$ area of induration and hyperpigmentation was noted in the right groin with no drainage. Skin examinations showed no other lesions. Her abdominal examination revealed no organomegaly and the remainder of the physical examination was normal.

The patient's white blood cell count was $5600 / \mathrm{mm}^{3}$, with $48 \%$ neutrophils, $44 \%$ lymphocytes, $5 \%$ monocytes, and $3 \%$ eosinophils. The hemoglobin was $9.6 \mathrm{~g} / \mathrm{dL}$, and the platelet count was $389,000 / \mathrm{mm}^{3}$. Aspartate aminotransferase and alanine aminotransferase were 12 and 24 units/L, respectively. The $\mathrm{C}$ reactive protein was $3.5 \mathrm{mg} / \mathrm{dl}$ and the erythrocyte sedimentation rate was $44 \mathrm{~mm} / \mathrm{h}$. Rapid plasma reagin and human immunodeficiency virus antibody by enzyme-linked immunosorbent assay were negative. A tuberculin skin test had $0 \mathrm{~mm}$ of induration. B. henselae immunoglobulin $\mathrm{M}$ and immunoglobulin $\mathrm{G}$ titers, by indirect florescent antibodies, were elevated at 1:32 and 1:512, respectively.

Skull radiography revealed that a focal irregular lucency involving the left frontal bone [Figure 1] and computed tomography of the head showed a focal soft-tissue lesion and a destructive process affecting the adjacent bone in the left frontal region. Magnetic resonance imaging of the head revealed septated scalp swelling with a destructive process involving adjacent calvaria with intracranial extension [Figure 2]. Pediatric neurosurgery performed a left frontal craniotomy and described purulent material on opening the skin, along with the erosion of the left frontal bone. Tissue microscopic examination showed chronic granulomatous tissue. The examination of the aspirate with a Warthin-Starry stain showed multiple clusters of organisms [Figure 3]. Aerobic, anaerobic, fungal, and mycobacterial culture were performed; a few colonies of Staphylococcus epidermidis grew in aerobic culture (considered a contaminant), and other cultures were negative. Finally, PCR from the skull abscess aspirate was positive for Bartonella species. No further speciation was performed, and culture for Bartonella culture was not attempted. The patient received 6-week treatment course with azithromycin and rifampin therapy with complete healing at the site of the infection.

\section{DISCUSSION}

Although rare, osteomyelitis is a known complication of CSD, with the vertebral column and pelvic girdle being the most commonly reported sites. ${ }^{[4]}$ Skull osteomyelitis has been reported previously as a complication of Bartonella infection, but is exceedingly rare. A literature review by Hajjaji et al. before 2007 found 47 reported cases of osteomyelitis due to $B$. henselae, with 4 of those involving the

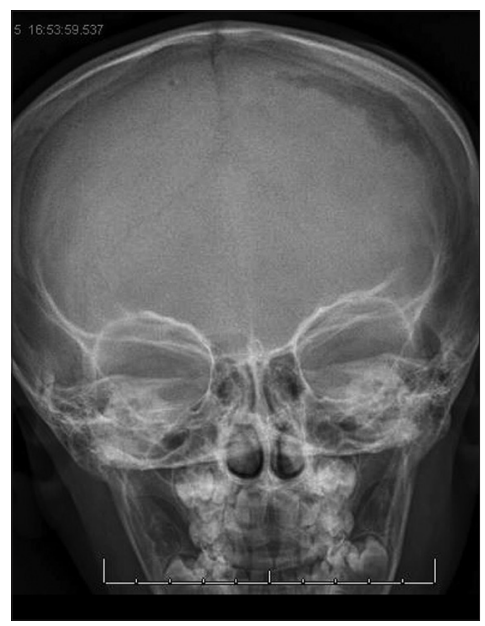

Figure 1: Skull X-ray showing lytic lesion in the left frontal bone (original)

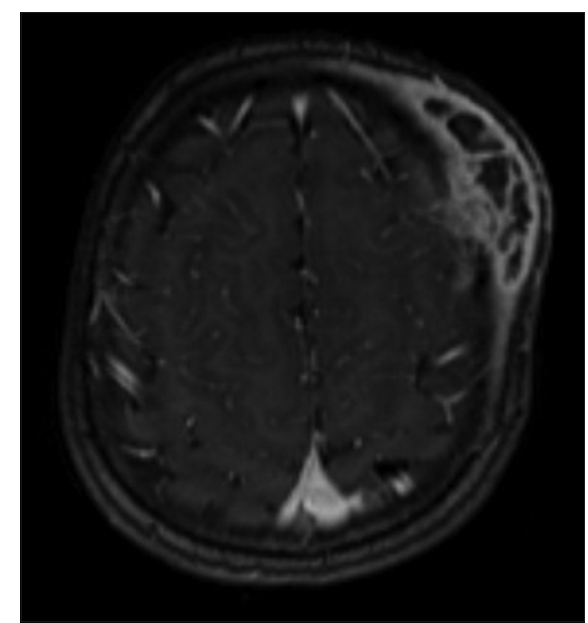

Figure 2: Septated scalp swelling measuring $5.5 \mathrm{~cm} \times 2.4 \mathrm{~cm} \times 1.7 \mathrm{~cm}$ within the left frontal region with a destructive process involving adjacent calvarium (original)

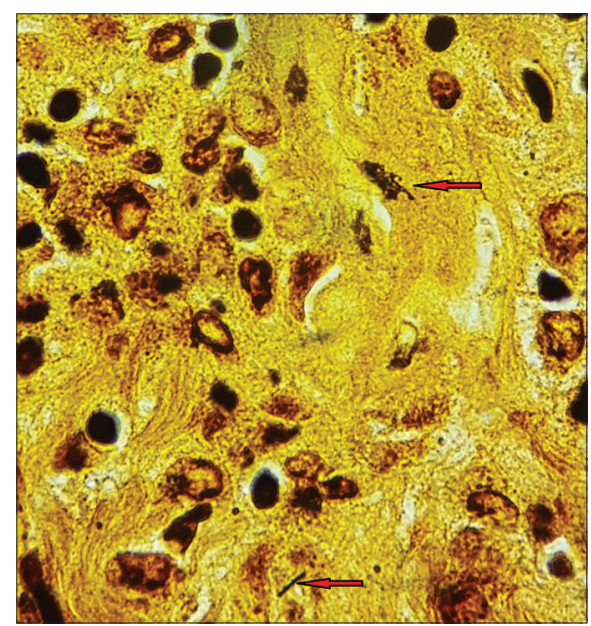

Figure 3: Warthin stain showing clumps of rod-shaped organisms (original)

skull. ${ }^{[4]}$ In this review, patients with Bartonella osteomyelitis usually had a subacute presentation with mild constitutional symptoms. Fever was present in $78 \%$ as part of their initial presentation, and most cases involved the axial skeleton. ${ }^{[4,5]}$ 
Since 2007, skull involvement was noted in just 1 of the 14 cases reported by Puri et al ${ }^{[5]}$ The frontal bone, parietal bone, and mastoid bone have all been reported to be involved. ${ }^{[4-6]}$ In addition, one other child presenting with lytic skull lesion involving the forehead after having cervical nodal disease has been reported. ${ }^{[7]}$ Overall, the clinical presentation of skull Bartonella osteomyelitis resembled that of Bartonella infection involving other bones. The skull infection had subacute or chronic course, associated lymph node disease, and need for debridement. ${ }^{[7]}$ Nevertheless, the reported long-term prognosis has been excellent, with complete recovery. ${ }^{[4,7]}$ Similarly, our patient had complete healing and recovery, consistent with the good outcome reported in other patients with Bartonella osteomyelitis.

The pathogenesis of Bartonella osteomyelitis is poorly understood, but both direct extension from infected lymph nodes (contiguous infection) and distant infection (presumably from hematogenous or lymphohematogenous spread) have been reported. ${ }^{[4,5,7]}$ Eight of the 14 cases reported by Puri et al. tested positive for Bartonella by PCR at the site of the infection. ${ }^{[5]}$ The hematogenous spread seems likely in our patient, who presented with inguinal and R-sided preauricular adenopathy and later developed osteomyelitis of the left frontal bone.

Spontaneous clinical improvement is expected in most cases of classical CSD. Thus, it is not surprising that the review by Hajjaji et al. concluded that some cases of Bartonella osteomyelitis have resolved despite treatment with antibiotics lacking activity against this organism. ${ }^{[4]} \mathrm{On}$ the other hand, the poor response to antibiotic treatment and the progression despite treatment in other reported cases suggests that therapy must be individualized. ${ }^{[7]}$ The role of antibiotic therapy of usually self-limited CSD in normal hosts is not well defined, while the evidence for benefit in immunocompromised patients with Bartonella infection is much stronger. Antibiotics that are considered effective against CSD include azithromycin, rifampin, ciprofloxacin, trimethoprim-sulfamethoxazole, and parenteral gentamicin.
In patients with a hepatosplenic CSD with prolonged fever, rifampin therapy reportedly has been effective. ${ }^{[8]}$ Gentamicin in combination with doxycycline is recommended as a treatment regimen for endocarditis. For musculoskeletal disease, most cases are managed with a combination of multiple courses of antibiotics and surgery. The optimal approach is yet to be determined. ${ }^{[4,7]}$

\section{CONCLUSION}

Our case highlights the importance of considering CSD in the differential of lytic skull lesions. Although rare, CSD may cause lytic skull lesions, which poses a diagnostic challenge, as immunologic, neoplastic, and other infectious etiologies must be ruled out.

\section{Financial support and sponsorship}

Nil.

\section{Conflicts of interest}

There are no conflicts of interest.

\section{REFERENCES}

1. Florin TA, Zaoutis TE, Zaoutis LB. Beyond cat scratch disease: Widening spectrum of Bartonella henselae infection. Pediatrics 2008;121:e1413-25.

2. Maman E, Bickels J, Ephros M, Paran D, Comaneshter D, Metzkor-Cotter E, et al. Musculoskeletal manifestations of cat scratch disease. Clin Infect Dis 2007;45:1535-40.

3. Raoult D. From cat scratch disease to Bartonella henselae infection. Clin Infect Dis 2007;45:1541-2.

4. Hajjaji N, Hocqueloux L, Kerdraon R, Bret L. Bone infection in cat-scratch disease: A review of the literature. J Infect 2007;54:417-21.

5. Puri K, Kreppel AJ, Schlaudecker EP. Bartonella osteomyelitis of the acetabulum: Case report and review of the literature. Vector Borne Zoonotic Dis 2015;15:463-7.

6. Muszynski MJ, Eppes S, Riley HD Jr. Granulomatous osteolytic lesion of the skull associated with cat-scratch disease. Pediatr Infect Dis J 1987;6:199-201.

7. Simonton K, Rupar D. Progressive cat scratch disease despite antimicrobial therapy. J Pediatric Infect Dis Soc 2015;4:e45-7.

8. Arisoy ES, Correa AG, Wagner ML, Kaplan SL. Hepatosplenic cat-scratch disease in children: Selected clinical features and treatment. Clin Infect Dis 1999;28:778-84. 